EL SALÓN DE EVENTOS "MACUILIS" EN VILLA OCUILTZAPOTLÁN, TABASCO: ¿AMPLIAR O FACTIBILIDAD DE RENOVARLO?

\title{
EL SALÓN DE EVENTOS “MACUILIS” EN VILLA OCUILTZAPOTLÁN, TABASCO: ¿AMPLIAR O FACTIBILIDAD DE RENOVARLO?
}

\section{"MACUILIS" EVENTS SALON FROM VILLA OCUILTZAPOTLÁN, TABASCO: EXPANDING OR RENEWING IT FEASIBILITY?}

Fabián Hernández Rodríguez*, Armando Mayo Castro**

\begin{abstract}
*Maestro en Administración. División Académica de Ciencias Económico Administrativas de la Universidad Juárez Autónoma de Tabasco.

**Doctor en Finanzas Públicas. División Académica de Ciencias Económico Administrativas de la Universidad Juárez Autónoma de Tabasco. Email: mayo_castro_armando@hotmail.com
\end{abstract}

Dirección para recibir correspondencia: fabrodriguez_85@hotmail.com 
EL SALÓN DE EVENTOS "MACUILIS" EN VILLA OCUILTZAPOTLÁN, TABASCO: ¿AMPLIAR O FACTIBILIDAD DE RENOVARLO?

\section{RESUMEN}

Dentro de los primeros 5 años, el Salón de Eventos Macuilis (2012-2016) con su fundadora la Mtra. Maribel Payró Avalos y su pequeño equipo de trabajo, han cubierto la necesidad de un espacio cómodo en un negocio rentable en la realización de eventos.

OBJETIVO: Incrementar el rendimiento y desarrollo del negocio mediante un estudio de factibilidad. El problema de un mejor espacio, con personal calificado, y en condiciones competitivas, es latente y preocupante. Analizando de esta manera la factibilidad de ampliación o renovación de la misma para brindar un mejor servicio a los clientes. La investigación es cuantitativa y cualitativa.

MATERIAL Y MÉTODO: Fue descriptivo y se realizó mediante las técnicas, de encuestas y entrevista. Esta fase de la investigación fue la más viable, para este tipo de estudio de mercado que permitió conocer de manera más analítica la situación con una mejor oportunidad de venta en el servicio de esta industria. El alcance es prospectivo, la profundidad descriptiva y el enfoque deductivo con la búsqueda documental adecuada, con un análisis financiero pertinente, y el impacto social deseado.

RESULTADOS: Revelaron la viabilidad de realizar un nuevo salón de eventos con mayor capacidad, sin dejar el salón actual que ha sido aceptado como necesario.

CONCLUSIONES: Se determinó que el proyecto es económicamente viable de llevarlo a cabo, puesto que, los indicadores de rentabilidad dieron como resultado un VAN positivo y una TIR redituable en tiempo estimado de inversión y rentabilidad.

PALABRAS CLAVE: Factibilidad. Operativo. Técnico. Financiero. 
EL SALÓN DE EVENTOS "MACUILIS" EN VILLA OCUILTZAPOTLÁN, TABASCO: ¿AMPLIAR O FACTIBILIDAD DE RENOVARLO?

\section{ABSTRACT}

Within the first five years, "Macuilis" events salon (2012-2016) with its founder Maribel Payró Ávalos and her small team, has covered the need of a comfortable place for the realization of events through a profitable business.

OBJECTIVE: To increase the efficiency and development of the business through a feasibility study. The need of a better space, with a more qualified personnel and more competitive conditions is latent and worrying. So, the feasibility for expanding or renewing the business to provide a better service to its customers has been analyzed through a quantitative and qualitative research.

MATERIAL AND METHOD: A descriptive method was used. It was carried out through survey and interview techniques. This was the most viable phase of the investigation, for this type of market research that allowed to know the situation in a more analytical way, and showed a better opportunity for selling services in this industry. Its scope was prospective with a descriptive depth and a deductive approach with an adequate documentary research, a pertinent financial analysis and the desired social impact.

RESULTS: I t was revealed the feasibility of creating a new events salon with a larger capacity, without leaving the current one, which has been considered as necessary.

CONCLUSIONS: It was determined that the project is economically viable to carry it out, since the profitability indicators resulted in a positive NPV and a profitable IRR in estimated time of investment and profitability.

KEY WORDS: Feasibility. Operative. Technical. Financial. 
EL SALÓN DE EVENTOS "MACUILIS" EN VILLA OCUILTZAPOTLÁN, TABASCO: ¿AMPLIAR O FACTIBILIDAD DE RENOVARLO?

\section{INTRODUCCIÓN}

\section{Antecedentes del salón de eventos Macuilis}

El Salón de eventos Macuilis, tuvo sus inicios el 23 de agosto de 2012, en Villa Ocuiltzapotlán Centro, Tabasco. El proyecto surgió por un matrimonio quien buscaba un lugar adecuado para posibles eventos sociales que tuviera un enfoque diferente a los demás, con un elegante modelo y diseño arquitectónico. La idea tuvo origen, por que en años anteriores la hija del matrimonio iba a cumplir sus XV años, rentando a su vez un casino social de la villa, llamado el Centro Social Rosa Magali, por ser el único que se contaba con mayor espacio para la realización del evento, pero no disponía con de las condiciones adecuadas para un buen festejo. Decidiendo después de cinco años crear este tipo de negocio rentable para el lugar y sus comunidades aledañas.

Salón de Eventos Macuilis es una microempresa formada por cuatro empleados, cada uno tiene diferentes actividades a realizar en tiempo y forma, de los cuales han desempeñado funciones de acuerdo a lo planeado antes, durante y después de un evento. Una de las funciones primordiales ha sido mantener las instalaciones en condiciones óptimas y en constante mantenimiento de manera diaria, pues en diversos casos después de las celebraciones se alteran algunos bienes muebles y el inmueble por parte de los invitados de algún festejo realizado, por lo que se requiere una exhaustiva vigilancia en el transcurso de los eventos por parte del administrador, así como en depreciación de algunos bienes muebles quienes deben sustituirse de manera inmediata para no generar problemas con alguna actividad que se realice dentro de las instalaciones, como los aires acondicionados, la iluminación, los baños, la entrada principal, las jardineras y el mantenimiento de paredes y los pisos para preservar la buena imagen de las instalaciones. Así como en mantener siempre un trato amable y de confianza con los clientes que realizan sus contratos en sus eventos, despejando sus dudas y también tomando en cuenta sus sugerencias.

En la siguiente figura del croquis se muestra la distribución (eventos en XV años, bodas, etc., y eventos infantiles para mejor organización del festejo. Actualmente, se maneja de acuerdo a la demanda del tipo de evento que se requiera para comodidad de los invitados (ver figura 1). 
EL SALÓN DE EVENTOS "MACUILIS" EN VILLA OCUILTZAPOTLÁN, TABASCO: ¿AMPLIAR O FACTIBILIDAD DE RENOVARLO?
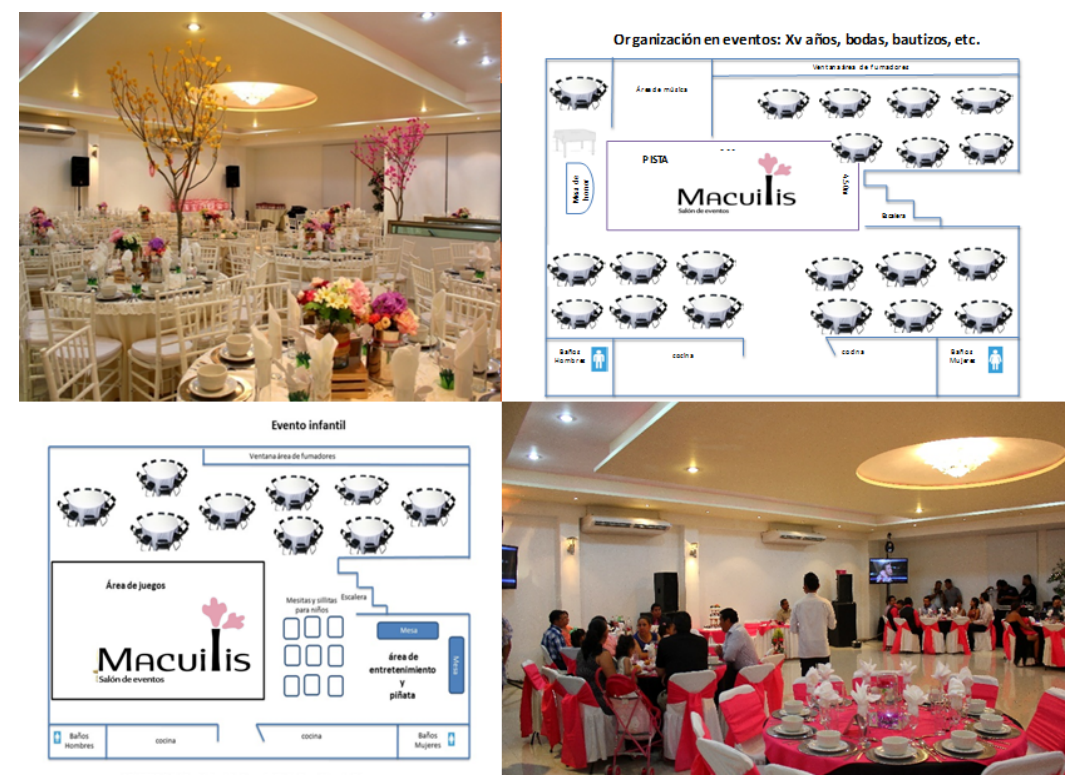

Figura 1. Croquis de distribución en tipo de eventos.

Fuente: Elaboración propia.

\section{Estructura organizativa. El desafío de la rentabilidad del salón Macuilis}

La estructura organizativa trabaja de manera interna las limitaciones de los niveles de mando en las funciones de cada personal antes, durante y después de cada evento, este proyecto cuenta en la actualidad con el dueño y el administrador del negocio encargado de planificar, organizar, controlar y dirigir las actividades propias del salón de eventos.

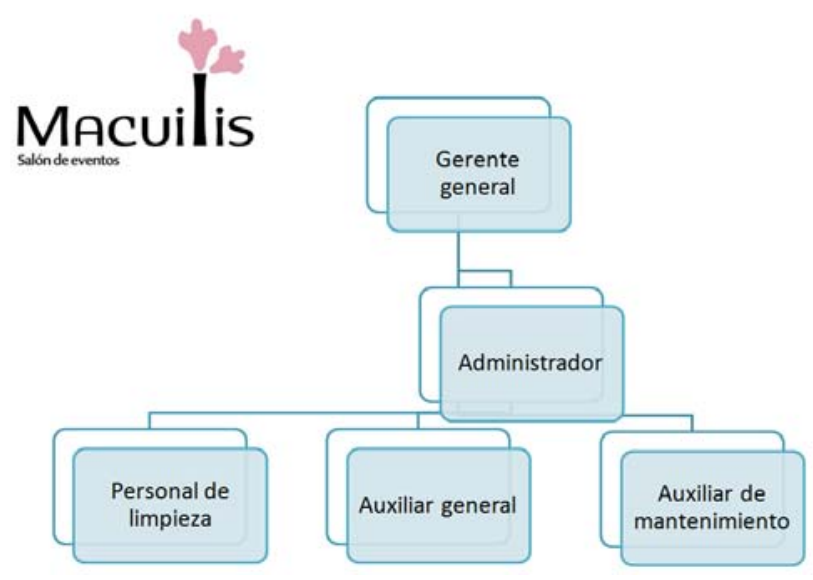

Figura 2. Organigrama salón de eventos Macuilis.

Fuente. Elaboración propia. 
EL SALÓN DE EVENTOS "MACUILIS" EN VILLA OCUILTZAPOTLÁN, TABASCO: ¿AMPLIAR O FACTIBILIDAD DE RENOVARLO?

\section{Planteamiento del problema}

Actualmente, el salón de eventos Macuilis, tiene una capacidad de 150 invitados, que se considera una demanda pertinente, desde su fundación, pero se ha observado que se necesita un salón con mayor capacidad para sus clientes, ya que también, son pocos los salones de eventos en Villa Ocuiltzapotlán que tienen el volumen adecuado para la realización de reuniones y festejos.

La demanda se ha incrementado en los primeros tres años principalmente en temporadas comunes como son los meses de febrero, abril, julio y diciembre, realizando hasta 12 eventos por mes en dichos periodos. En los meses que se han contado pocos contratos son enero, marzo y octubre de 6 eventos mensualmente. Actualmente, no se cuenta con un estacionamiento propio por lo es una debilidad en el negocio y en el servicio a prestar.

\section{OBJETIVOS}

General:

Realizar un estudio de factibilidad para la creación de un nuevo salón de eventos, que proporcione información suficiente y confiable a los actuales y futuros inversionistas del salón de eventos Macuilis.

\section{Específicos:}

- Estudio de factibilidad financiera como estrategia de crecimiento de los actuales y futuros inversionistas del salón de eventos Macuilis.

- Investigación de mercado en la Villa Ocuiltzapotlán, Villa Macultepec y comunidades aledañas para determinar la oferta y demanda en negocios de eventos sociales.

- Determinar el monto de inversión necesario, los costos para el desarrollo e implementación del servicio y el tiempo que tomará recuperar la inversión.

\section{Justificación}

El presente estudio pretende crear un referente de empresa de calidad, variedad, innovación y organizar eventos, ahorrando el tiempo de los clientes y ampliando las oportunidades de 
EL SALÓN DE EVENTOS "MACUILIS" EN VILLA OCUILTZAPOTLÁN, TABASCO: ¿AMPLIAR O FACTIBILIDAD DE RENOVARLO?

negocios de los proveedores. El reto de este estudio de factibilidad es, conocer si es viable la apertura de un nuevo salón de eventos con mayor capacidad para comodidad de los futuros clientes, con el fin de conocer si es oportuna la inversión que se pretende realizar con el método de estudio financiero.

\section{MATERIAL Y MÉTODO}

La investigación descriptiva será utilizada mediante el método por encuestas y entrevistas.

Esta fase de la investigación es la más adecuada para este tipo de estudio de mercado puesto que me permitirá establecer de manera más detallada la situación del mercado de esta industria y me proporcionara mayor cantidad de herramientas que permiten proyectar aspectos muy importantes a la hora de realizar la investigación de campo.

Será la técnica principal que se utilizará dentro del proyecto para obtener la información necesaria de los clientes potenciales, así como de la competencia.

Una de las informaciones más importantes que necesito extraer son las preferencias de las personas en cuanto a gustos precios y presentaciones, esta información que me permita saber las principales necesidades de nuestros futuros consumidores.

Actores a entrevistar

- Periodo de recolección de la información de campo: Marzo de 2017.

- Población de la muestra: hombres y mujeres mayores de 25 años.

- Tamaño de la muestra: 55 encuestas.

- Técnica de recolección de los datos: Personal.

- Método de procesamiento de los datos: Word.

- Fecha del reporte: 31 de Marzo de 2017.

- Método de aplicación: Escala de Likert.

Actores a entrevistar: se realizó a clientes que han rentado en diversas ocasiones el actual salón Macuilis con diferentes tipos de eventos ya que es un estudio fundamental en el servicio 
EL SALÓN DE EVENTOS "MACUILIS" EN VILLA OCUILTZAPOTLÁN, TABASCO: ¿AMPLIAR O FACTIBILIDAD DE RENOVARLO?

prestado y así conocer sus puntos de vista de cada uno en el futuro proyecto de inversión de un nuevo salón de eventos que se pretende implementar.

A continuación, se relata el resultado de encuesta más significativo.

El primer estudio realizado por método de la Escala de Likert en el servicio prestado para las los clientes en sus eventos determino que el $11.11 \%$ es regular, el 11.11 muy buena y finalmente, el 77.78 es buena, por lo que la percepción es aceptable para los clientes en los eventos que han efectuado, asegurando que rentarían nuevamente el salón cuando esta se requiera, tal y como se muestra en la siguiente gráfica (ver figura 3).

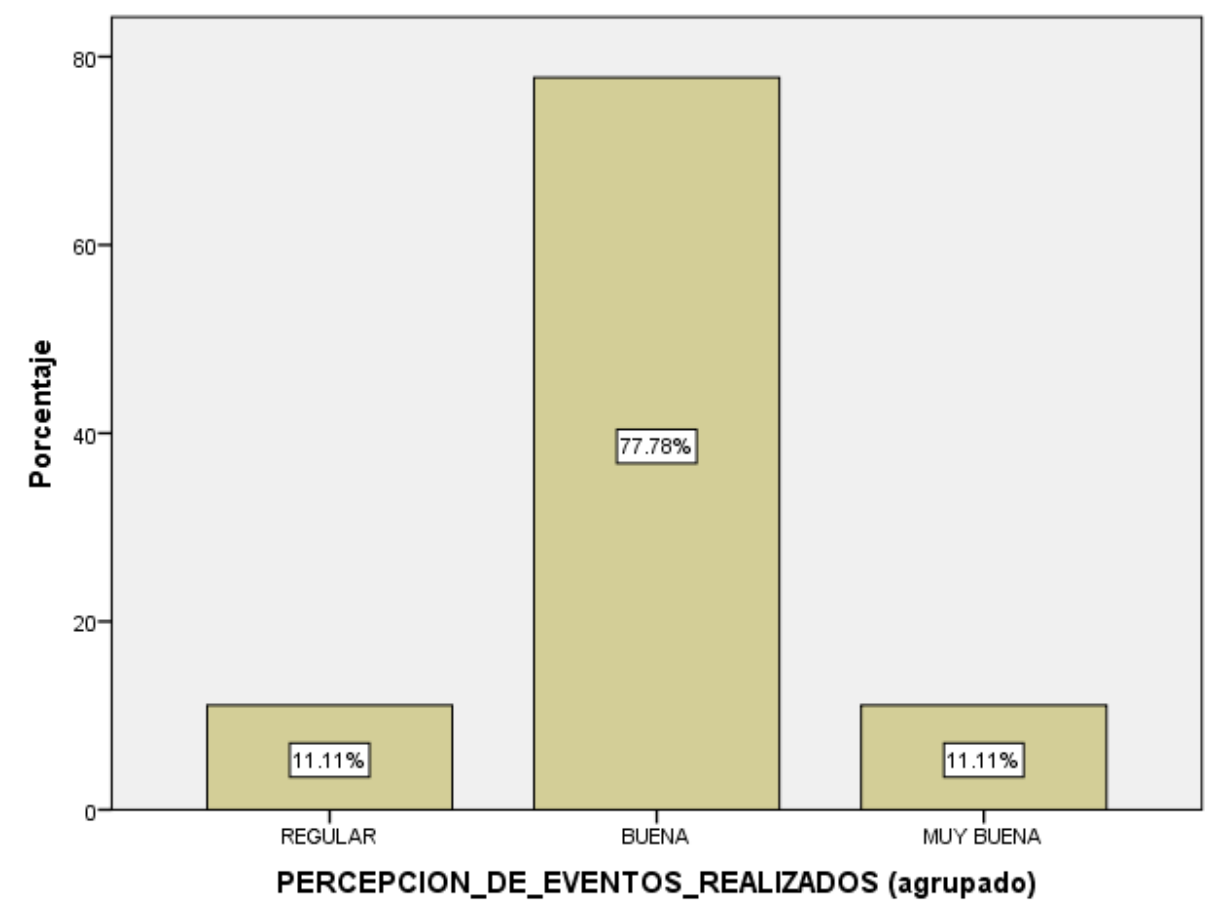

Figura 3. Percepción de eventos realizados (agrupado).

Fuente: Elaboración propia.

El segundo análisis de acuerdo a la iniciativa del nuevo proyecto a emprender en el estudio de Escala de Likert en las entrevistas realizadas a los clientes, arrojo que el $44.44 \%$ consideró buena la idea de implementar un nuevo salón de eventos con mayor capacidad, por lo tanto el $55.56 \%$ estimó de igual forma muy buena la idea de una mayor renovación del salón de eventos en su ampliación para mejora de los servicios futuros realizar. 
EL SALÓN DE EVENTOS "MACUILIS" EN VILLA OCUILTZAPOTLÁN, TABASCO: ¿AMPLIAR O FACTIBILIDAD DE RENOVARLO?

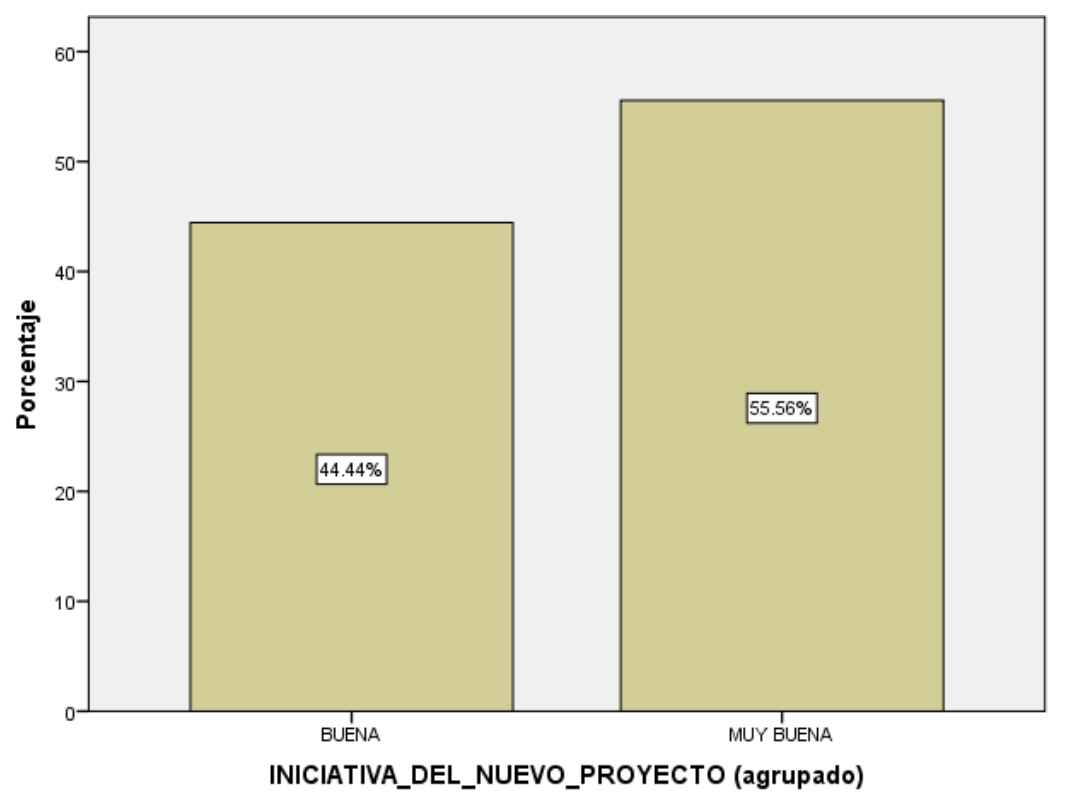

Figura 4. Iniciativa del nuevo proyecto a emprender (agrupado).

Fuente: Elaboración propia.

\section{Análisis Financiero}

\section{Inversión inicial}

La inversión requerida antes de la puesta en marcha debe tomarse en cuenta valores requeridos para poder invertir, desde el punto de vista de lo tangible o intangible de estos valores. Estas cifras se muestran en las siguientes tablas clasificados en: Equipo de oficina y cómputo, equipo de transporte, servicios, recursos humanos y entre otros.

Tabla 1

Estimación de inversión inicial en 2017

\begin{tabular}{|c|c|c|c|}
\hline Inversión fija & Cantidad & Costo unitario & Costo \\
\hline \multicolumn{4}{|l|}{ Mobiliario y equipo } \\
\hline Escritorio & 2 & $\$ 2,765.00$ & $\$ 5,530.00$ \\
\hline Archivero & 1 & $\$ 1,200.00$ & $\$ 1,200.00$ \\
\hline Sillas & 5 & $\$ 765.00$ & $\$ 3,825.00$ \\
\hline Cestos & 2 & $\$ 123.00$ & $\$ 246.00$ \\
\hline
\end{tabular}


EL SALÓN DE EVENTOS "MACUILIS" EN VILLA OCUILTZAPOTLÁN, TABASCO: ¿AMPLIAR O FACTIBILIDAD DE RENOVARLO?

\begin{tabular}{|c|c|c|c|}
\hline Inversión fija & Cantidad & Costo unitario & Costo \\
\hline \multicolumn{4}{|l|}{ Equipo de cómputo } \\
\hline Computadora & 1 & $\$ 19,000.00$ & $\$ 19,000.00$ \\
\hline Impresora & 1 & $\$ 5,432.00$ & $\$ 5,432.00$ \\
\hline \multicolumn{4}{|l|}{ Equipo de transporte } \\
\hline Automóvil camioneta estaquita & 1 & $\$ 235,400.00$ & $\$ 235,400.00$ \\
\hline \multicolumn{4}{|l|}{ Muebles } \\
\hline Mesas redondas & 30 & $\$ 543.00$ & $\$ 16,290.00$ \\
\hline Mesas tablón & 5 & $\$ 453.00$ & $\$ 2,265.00$ \\
\hline Sillas plegables & 310 & $\$ 132.00$ & $\$ 40,920.00$ \\
\hline Sillas infantiles pegables & 100 & $\$ 78.00$ & $\$ 78.00$ \\
\hline Mesitas rectangulares infantiles & 10 & $\$ 123.00$ & $\$ 123.00$ \\
\hline Juego inflable & 1 & $\$ 12,000.00$ & $\$ 12,000.00$ \\
\hline Nevera & 2 & $\$ 2,342.00$ & $\$ 4,684.00$ \\
\hline Total de inversión Fija & & & $\$ 348,100.00$ \\
\hline \multicolumn{4}{|l|}{ Costos directos de operación } \\
\hline \multicolumn{4}{|l|}{ Costos indirectos } \\
\hline \multicolumn{4}{|l|}{ Inversión diferida } \\
\hline Organización y constitución del salón & 1 & $\$ 5,000.00$ & $\$ 5,000.00$ \\
\hline Construcción de predio & 1 & $\$ 498,522.10$ & $\$ 498,522.10$ \\
\hline Contrato de teléfono e internet & 1 & $\$ 631.00$ & $\$ 631.00$ \\
\hline \multicolumn{4}{|l|}{ Capital de trabajo } \\
\hline Caja & 1 & $\$ 8,000.00$ & $\$ 8,000.00$ \\
\hline Bancos & 1 & $\$ 25,000.00$ & $\$ 25,000.00$ \\
\hline Total de inversión diferida & & & $\$ 537,153.10$ \\
\hline Total de inversión & & & $\$ 885,253.10$ \\
\hline
\end{tabular}


EL SALÓN DE EVENTOS "MACUILIS" EN VILLA OCUILTZAPOTLÁN, TABASCO: ¿AMPLIAR O FACTIBILIDAD DE RENOVARLO?

\begin{tabular}{lccc}
\hline \multicolumn{1}{c}{ Inversión fija } & Cantidad & Costo unitario & Costo \\
\hline Financiamientos & & \\
Inversiones de socios & $40 \%$ & $\$ 230,911.10$ \\
Aportaciones de bancos & $60 \%$ & $\$ 654,342.00$ \\
\hline
\end{tabular}

Fuente: Elaboración propia.

Se determinó proyectar los ingresos a 5 años, para poder contar con el margen de ganancias que se percibirán por los eventos anualmente.

Tabla 2

Estimación de ingresos en cinco años

\begin{tabular}{crrr}
\hline Año & \multicolumn{2}{l}{ Ingresos } & Porcentaje ganancia estimado \\
\hline 2017 & $\$$ & $490,000.00$ & \\
2018 & $\$$ & $539,000.00$ & $10 \%$ \\
2019 & $\$$ & $619,850.00$ & $15 \%$ \\
2020 & $\$$ & $743,820.00$ & $20 \%$ \\
2021 & $\$$ & $892,584.00$ & $20 \%$ \\
Total ingresos & $\$$ & $3,285,254.00$ & \\
\hline
\end{tabular}

Fuente: Elaboración propia.

\section{Presupuesto mensual 2017}

De acuerdo a la siguiente tabla se determinó el cálculo de los ingresos y egresos que se obtienen dentro de los periodos mensuales en base a los resultados financieros, se conoce la viabilidad del proyecto a emprender, tomando en cuenta el estimado de ampliación y puesta en marcha de la empresa y su proyección en cinco años y se muestra en las siguientes tablas. 
Original

EL SALÓN DE EVENTOS "MACUILIS" EN VILLA OCUILTZAPOTLÁN, TABASCO: ¿AMPLIAR O FACTIBILIDAD DE RENOVARLO?

Tabla 3

Presupuesto mensual en 2017

\begin{tabular}{|c|c|c|c|c|c|c|c|c|c|c|c|c|c|}
\hline Ingresos & Enero & Febrero & Marzo & Abril & Mayo & Junio & Julio & Agosto & Septiembre & Octubre & Noviembre & Diciembre & Saldo \\
\hline Ingreso inicial & $\$ 885,353.10$ & & & & & & & & & & & & \\
\hline $\begin{array}{l}\text { Ingresos } \\
\text { Eventos } \\
\text { mensuales }\end{array}$ & & & & & & & & & & & & & $\$$ \\
\hline $\begin{array}{l}\text { Total de } \\
\text { ingresos }\end{array}$ & $\$ 60,000.00$ & $\$ 70,654.00$ & $\$ 60,876.00$ & $\$ 54,648.10$ & $\$ 77,679.00$ & $\$ 55,763.00$ & $\$ 60,874.00$ & $\$ 45,987.00$ & $\$ 65,483.00$ & $\$ 60,763.00$ & $\$ 92,543.00$ & $\$ 98,654.00$ & $\$ 803,924.10$ \\
\hline $\begin{array}{l}\text { Egresos } \\
\text { Servicios }\end{array}$ & & & & & & & & & & & & & \\
\hline $\begin{array}{l}\text { Básicos } \\
\text { Proyectado }\end{array}$ & $\$ 1,550.00$ & $\$ 1,550.00$ & $\$ 1,550.00$ & $\$ 1,550.00$ & $\$ 1,550.00$ & $\$ 1,550.00$ & $\$ 1,550.00$ & $\$ 1,550.00$ & $\$ 1,550.00$ & $\$ 1,550.00$ & $\$ 1,550.00$ & $\$ 1,550.00$ & $\$ 18,600.00$ \\
\hline $\begin{array}{l}\text { Gastos de } \\
\text { operación }\end{array}$ & $\$ 5,249.00$ & $\$ 5,249.00$ & $\$ 4,325.00$ & $\$ 4,355.00$ & $\$ 3,452.00$ & $\$ 4,326.00$ & $\$ 4,573.00$ & $\$ 3,321.00$ & $\$ 3,242.00$ & $\$ 3,211.00$ & $\$ 5,249.00$ & $\$ 5,249.00$ & $\$ 51,801.00$ \\
\hline $\begin{array}{l}\text { Gastos de } \\
\text { administración }\end{array}$ & $\$ 14,100.00$ & $\$ 14,100.00$ & $\$ 14,100.00$ & $\$ 13,100.00$ & $\$ 13.10$ & $\$ 12,121.00$ & $\$ 9,876.00$ & $\$ 1,321.00$ & $\$ 1,211.00$ & $\$ 12,342.00$ & $\$ 14,563.00$ & $\$ 16,754.00$ & $\$ 123,601.10$ \\
\hline $\begin{array}{l}\text { Gastos de } \\
\text { venta }\end{array}$ & $\$ 7,299.00$ & $\$ 5,425.00$ & $\$ 4,245.00$ & $\$ 3,251.00$ & $\$ 5,563.00$ & $\$ 6,252.00$ & $\$ 4,232.00$ & $\$ 4,325.00$ & $\$ 3,142.00$ & $\$ 5,252.00$ & $\$ 7,299.00$ & $\$ 7,299.00$ & $\$ 63,584.00$ \\
\hline Total Egresos & $\$ 28,198.00$ & $\$ 26,324.00$ & $\$ 24,220.00$ & $\$ 22,256.00$ & $\$ 10,578.10$ & $\$ 24,249.00$ & $\$ 20,231.00$ & $\$ 10,517.00$ & $\$ 9,145.00$ & $\$ 22,661.00$ & $\$ 28,661.00$ & $\$ 30,852.00$ & $\$ 257,586.10$ \\
\hline $\begin{array}{l}\text { Flujos netos de } \\
\text { efectivo }\end{array}$ & $\$ 31,802.00$ & $\$ 44,330.00$ & $\$ 36,656.00$ & $\$ 32,392.10$ & $\$ 67,100.90$ & $\$ 31,514.00$ & $\$ 40,643.00$ & $\$ 35,470.00$ & $\$ 56,338.00$ & $\$ 63,882.00$ & $\$ 63,882.00$ & $\$ 67,802.00$ & $\$ 490,000.00$ \\
\hline
\end{tabular}

Fuente: Elaboración propia. 
EL SALÓN DE EVENTOS "MACUILIS" EN VILLA OCUILTZAPOTLÁN, TABASCO: ¿AMPLIAR O FACTIBILIDAD DE RENOVARLO?

Tabla 4

Proyección de inversión en cinco años

\begin{tabular}{lccccc}
\hline Estimado & 2017 & 2018 & 2019 & 2020 & 2021 \\
\hline Inflación & $10 \%$ & $15 \%$ & $20 \%$ & $20 \%$ \\
FNE & $\$ 490,000.00$ & $\$ 539,000.00$ & $\$ 619,850.00$ & $\$ 743,820.00$ & $\$ 892,584.00$ \\
\hline
\end{tabular}

Fuente: Elaboración propia.

\section{Discusión}

El Salón Macuilis se interesa en conocer como primer punto, la factibilidad de creación de un nuevo salón de eventos para implementar, de acuerdo a los resultados, en gustos y necesidades de los clientes y habitantes de la Villa un lugar adecuado y seguro para los que demanden sus eventos y reuniones; en segundo punto, se busca incrementar el número de eventos que demanden las personas en la Villa y sus comunidades aledañas; como tercer punto, se examina en tener la oportunidad de incrementar la demanda no solo en la villa, buscando en promoverse en la ciudad de Villahermosa para aumentar la demanda en los servicios de renta; como cuarto punto la empresa busca el desarrollo económico de la zona, atrayendo inversión que incremente el bienestar, calidad de vida y beneficio a los habitantes de la villa, es por ello que el quinto punto está ligado con el anterior ya que en ello se busca crear empleos permanentes que proporcionen bienestar a las personas relacionadas directa e indirectamente con la empresa; como penúltimo punto, conocer si la rentabilidad del salón será factible en un mediano y largo plazo y si su inversión puede generar los resultados óptimos para los inversionistas; Por último, analiza la estimación de una proyección de ventas factible con una utilidad neta anual de 5 años en su recuperación de inversión. Los precios de los servicios deberán cubrir los costos y permitir un margen de utilidad, pero buscando que sean competitivos en el mercado, teniendo en cuenta que son eventos ocasionales, y de acuerdo a los servicios que el cliente seleccione, las opciones que escoja y al número de invitados, puede variar el valor total de su evento, poniendo a disposición el servicio de contrato del salón con asesoramiento y acompañamiento continuo, buscando se identificados por la calidad, innovación y elegancia. 
EL SALÓN DE EVENTOS "MACUILIS" EN VILLA OCUILTZAPOTLÁN, TABASCO: ¿AMPLIAR O FACTIBILIDAD DE RENOVARLO?

\section{CONCLUSIONES}

Realizado el análisis del estudio de factibilidad en la creación de un nuevo salón de eventos del salón Macuilis, se puede concluir en lo siguiente:

- De acuerdo lo planteado en la investigación en el manejo de negocio de eventos sociales que satisfaga las exigencias de los clientes potenciales, se pudo constatar mediante la investigación de mercado que es viable crear en Villa Ocuiltzapotlán, Centro, Tabasco, la creación de un nuevo salón de eventos, sin dejar sus funciones el actual salón de fiestas Macuilis, puesto que los resultados reflejaron la disposición del mercado objetivo de demandar nuestros servicios.

- En la sustentabilidad legal y constitución de socios de las actividades empresariales lo ideal es que la empresa se constituya como una Compañía de Responsabilidad Limitada, pues esta forma jurídica presenta una serie de ventajas, como ejemplo: los socios en caso de quiebra no arriesgan el patrimonio personal, pues solamente responden por el monto de sus aportaciones individuales, para disolver la compañía se requiere la aprobación de cada uno de los socios. En sí, su constitución es ágil y segura, pero para ello es de suma importancia cumplir con los requisitos, leyes y reglamentos.

- Con base al estudio financiero se determinó que el proyecto es económicamente viable de llevarlo a cabo, puesto que, los indicadores de rentabilidad dieron como resultado un VAN positivo y una TIR redituable en tiempo estimado de inversión y rentabilidad.

- Como último punto se concluye que organizar un evento cual fuese su tipo no es una tarea sencilla, pues su éxito depende de una apropiada planificación, coordinación y control de cada una de las actividades, las mismas que tienen que ser desarrolladas por un equipo de trabajo con capacidad, experiencia y conocimiento en el área, por lo tanto se dedicara la mayor responsabilidad y satisfacción hacia los clientes. 
EL SALÓN DE EVENTOS "MACUILIS" EN VILLA OCUILTZAPOTLÁN, TABASCO: ¿AMPLIAR O FACTIBILIDAD DE RENOVARLO?

\section{REFERENCIAS BIBLIOGRÁFICAS}

Alvarado P. (2011). Estudio de Factibilidad para la instalacion de un salon de eventos en la ciudad de Cuenca (Tesis). Universidad Ecuador.

APA, N. (s.f.). Centro de escritura Javeriano. Normas APA, Sexta edición.

Hoyos R. (2016). Plan de Marketing, diseño implementacion y control. Ecoe ediciones.

Baca G. (2001). Evaluacion de proyectos. Mexico: McGraw-Hill.

Cariola. (2009). Plan para emprendedores. Buenos Aires: Nobuko.

Ruiz Y. (2016). Plan de Negocios para la construccion de un salon de eventos en la ciudad de Neiva, Bogota. Espacialidad en Gerencias de empresas Monografía. Fundacion Universidad de América. Bogotá, Colombia.

Bernal C. (2010). Metodologia de la Investigación. Colombia: Pearson Educación.

Drudis A. (s.f.). Planificacion, organizacion y gestion de proyectos. Barcelona: Gestiones 2000.

Ebook (2016). 10 ejemplos de factibilidad y estudio de mercado, Guia para tu tesis.

Errosa M. E. (1999). Proyectos de inversion en ingenieria, Metodología. México: Limusa.

Garcia J. (2016). Estrategia de Inversion y rentabilidad. Emprende Pyme.

Guzman C. (2006). Matematicas financieras para la toma de decisiones. Edición electrónica. Recuperado de http://www.adizesca.com/site/assets/mematematicas_financieras_para_toma_de_decisiones_empresariales-ca.pdf

$H$ ayuntamiento reglamento, m. d. (s.f.). Licencias y reglamentos del $H$, ayuntamiento del Centro, Tab. licencias, uso de suelo, edificacion, permisos. Villahermosa, Tabasco, Mexico.

García J., Paredes L. (2004). Estrategias financieras empresariales. México: Ebook. 